\title{
ESTIMACION VISCOSA EN SISTEMAS ELÁSTICOS GENERALIZADOS
}

Fecha de recepción: 25 de marzo de 2014 • Fecha de aceptación: 21 de mayo de 2014

\section{VISCOSITY ESTIMATION IN ELASTIC SYSTEMS GENERALIZED}

Adrian Ricardo Gómez Plata

\section{RESUMEN}

En la teoría de Ecuaciones Diferenciales parciales, se tiene un campo muy prolífico y novedoso de la solución de ecuaciones que respetan ciertos criterios de conservación y balance, conocido como leyes de conservación hiperbólica. En esta área se usan métodos como el de las regiones invariantes, compacidad compensada y, por supuesto, el principio del máximo. En el presente trabajo se usará esta última metodología para encontrar estimaciones suaves clásicas de los conocidos sistemas de elasticidad. Así mismo, se presenta la solución a sistemas elásticos generalizados con condiciones acotadas medibles, encontrándose estimaciones de las soluciones viscosas globales suaves en el sentido clásico para este sistema usando el principio del máximo.

Palabras clave: Leyes de conservación hiperbólica, principio del máximo, ecuaciones diferenciales parabólicas, problema de Cauchy, estimaciones viscosas.

\section{ABSTRACT}

In the theory of partial differential equations, it has a very prolific and innovative field of solving equations that respect certain standards of conservation and balance, known as hyperbolic conservation laws. In this area, methods such as invariant regions, compensated compactness and off course the maximum principle are used. In this paper, the latter method is used for finding classical soft estimates of known elastic systems. Moreover, the estimation of viscosity solutions in elasticity generalized systems is presented with bounded measurable conditions, finding a priori estimations of soft global viscous solutions of the system using the maximum principle.

Keywords: Hyperbolic conservations Laws, maximum principle, cauchy's problems, viscous estimations.

1. M.Sc- EAFIT-Col, Ph.D(c) en Matemática Aplicada-UNICAMP-Br. Profesor Departamento de Matemáticas, Facultad de Ciencias Básicas y Aplicadas, Universidad Militar Nueva Granada. e-mail: adrian.gomez@unimilitar.edu.co 


\section{INTRODUCCIÓN}

Los científicos ingleses Hooke y Newton desde el siglo XVII empezaron a forjar nuevas ideas en sus mediciones experimentales, en especial Hooke conceptualizo la noción de elasticidad para los resortes en 1660, describiendo cómo un cuerpo elástico se estira de forma proporcional a la fuerza que se ejerce sobre él, lo que dio lugar a la invención del resorte helicoidal o mejor conocido como muelle.

En la actualidad el estudio de los sistemas de elasticidad puede realizarse desde diversas áreas de la ciencia, e ingeniería. Los ingenieros civiles están interesados en que sus estructuras de concreto mantengan niveles de elasticidad que toleren fenómenos sísmicos, los físicos les interesa tener en cuenta la elasticidad para el estudio de la resistencia de un material, algunos especialistas en física analizan la elasticidad de los fluidos y de ciertos gases. La elasticidad de un material es definida en ingeniería y física como la relación que se da entre la fatiga y la deformación de un objeto. La elasticidad finita es la teoría del estudio de las grandes deformaciones en los objetos, esta teoría es no lineal y por ende una teoría de difícil tratamiento matemático y físico.

En general las teorías de la elasticidad se han convertido en una área de estudio desde la matemática, debido a su difícil entendimiento como objeto físico, de hecho en libros como el de Gurtin, 1981, y Ciarlet, 1989, se hace un estudio interesante de la elasticidad finita y de la elasticidad en tres dimensiones respectivamente, donde el componente matemático es fundamental para dar interpretaciones y argumentaciones al interior de la teoría.

En este trabajo se aborda el estudio de las soluciones de un sistema general de ecuaciones de elasticidad, que son conocidos como sistemas de elasticidad extendidos con fuente. Según Lu, 2002, este tipo de sistemas generalizan los sistemas de ecuaciones diferenciales parciales relacionados a un gas politrópico, sistemas especiales de ecuaciones de Euler para un fluido compresible. El estudio está relacionado a las Ecuaciones diferenciales Parciales, en particular a un tópico especial Ilamado Leyes de conservación hiperbólica. En este artículo se dan estimaciones de las soluciones viscosas a un sistema de elasticidad que presentan conservación de masa-momento.

En el sentido clásico de la física un problema elástico queda definido por la geometría del cuerpo antes de ser deformado, las fuerzas exteriores, que dan lugar al término "potencial" del lagrangiano y las componentes del tensor de constantes elásticas.

Un lagrangiano según Landau y Lifshits, 1985, es una función matemática a partir de la cual se pueden derivar las leyes de conservación y otras propiedades importantes de un sistema físico. De hecho, en física moderna el lagrangiano se considera el objeto más fundamental que describe un sistema físico.

En matemáticas esta idea de elasticidad se generaliza por medio de una ley de conservación Hiperbólica, que es un sistema de ecuaciones quasilineal de la forma

$$
u_{t}+f(u)_{x}=0, \text { con }(x, t) \in R \times R^{+}
$$

donde $u=\left(u_{1}, \ldots, u_{n}\right)^{\top} \in R^{n}, n \geq 1$, es una función vectorial desconocida, que modela una cantidad física y $f\left(f\left(u_{1}\right), \ldots, f\left(u_{n}\right)\right)^{\top}$ es un vector dado que denota un término de conservación asociado generalmente a dicha cantidad física.

Un ejemplo de este sistema físico y de conservación es

$$
\left\{\begin{array}{l}
u_{t}+\left(c u+f(v)_{x}\right)+g_{1}(u, v)=0 \\
v_{t}+(u+g(v))_{x}+g_{2}(u, v)=0
\end{array}\right.
$$

con

$$
(u(x, 0), v(x, 0))=\left(u_{0}(x), v_{0}(x)\right)
$$




\section{Evans, 1998, hace una clara introducción a las ecuaciones parabólicas, operadores parabólicos y al principio del máximo débil y fuerte, en particular Protter y Weinberger, 1999, estudian el principio del máximo para ecuaciones diferenciales ordinarias para pasar al principio del máximo para ecuaciones diferenciales parciales parabólicas. Landis, 1997, desarrolla tópicos matemáticos avanzados para esta teoría.}

donde $\mathbf{C}$ es una constante, $\mathbf{V}$ es la deformación, $\mathbf{f}(\mathbf{v})$ es la fuerza, $\mathbf{u}$ es la velocidad y $\boldsymbol{g}_{\mathbf{1}}$, $\mathrm{g}_{2}$ son términos fuente del sistema.

Lu 2002, estudia sistemas de conservación de elasticidad Lagrangiana en una dimensión no lineal dado por

$$
\left\{\begin{array}{l}
u_{t}+f(v)_{x}=0 \\
u_{t}+u_{x}=0
\end{array}\right.
$$

con condiciones acotadas medibles

$$
(\mathrm{u}(\mathrm{x}, 0), \mathrm{v}(\mathrm{x}, 0))=\left(\mathrm{u}_{0}(\mathrm{x}), \mathrm{v}_{0}(\mathrm{x})\right)
$$

donde $\mathbf{V}$ es la deformación, $\mathbf{f}(\mathbf{V})$ es la fuerza y u la velocidad.

El anterior sistema es un caso particular de (1) y lo que se hace en este trabajo es generalizarlo y encontrar estimaciones clásicas de su solución.

En este sentido se abordará la búsqueda de estimaciones viscosas de sistemas de elasticidad que generaliza el caso de los sistemas de elasticidad Lagrangianos. En particular demostraremos usando el principio del máximo para ecuaciones diferenciales parabólicas la existencia de estimaciones de las soluciones viscosas globales suaves de (1).

\section{TEORÍA Y MÉTODOS MATEMÁTICOS}

\section{Principio del Maximo-Leyes de \\ Conservacion Hiperbolica}

Evans, 1998, hace una clara introducción a las ecuaciones parabólicas, operadores parabólicos y al principio del máximo débil y fuerte, en particular Protter y Weinberger, 1999, estudian el principio del máximo para ecuaciones diferenciales ordinarias para pasar al principio del máximo para ecuaciones diferenciales parciales parabólicas. Landis, 1997, desarrolla tópicos matemáticos avanzados para esta teoría.

Lu, 2002, hace una breve y precisa introducción a los sistemas de leyes de conservación y define los invariantes de Riemann. Yan el al., 2007, demuestran cómo usar el principio del máximo en sistemas de leyes de conservación hipérbolica.

\section{Cálculo de estimaciones a priori}

Lu, 2002, usando el método de las regiones invariantes y Gómez-Plata, 2009, usando el principio del máximo, garantizan la existencia de soluciones viscosas globales del sistema (1) sin los términos fuentes $g_{1}(u, v), g_{2}(u, v)$, usando una regularización 
del sistema, como lo indica los términos a la derecha del sistema

$$
\left\{\begin{array}{l}
u_{t}+(c u+f(v))_{x}=\varepsilon u_{x x} \\
v_{t}+(u+g(v))_{x}=\varepsilon v_{x x}
\end{array}\right.
$$

y cuyas condiciones acotadas medibles son las mismas enunciadas en (1).

En analogía al método usado por Gómez-Plata, 2009, se regulariza y encuentra estimaciones viscosas de las soluciones del sistema (1) usando el principio del máximo para el sistema de elasticidad generalizado dado por

$$
\left\{\begin{array}{l}
u_{t}+\left(c u+f(v)_{x}\right)+g_{1}(u, v)=\varepsilon u_{x x} \\
v_{t}+(u+g(v))_{x}+g_{2}(u, v)=\varepsilon v_{x x}
\end{array}\right.
$$

\section{RESULTADO}

\section{Estimaciones usando el Principio del Máximo}

Consideremos el sistema (3) con las condiciones acotadas medibles enunciadas en (1) y suponiendo, para $\mathbf{f}, \mathbf{g}$ que:

A. $g(v)$ y $f(v)$ cumplen que $f, g \in C^{3}$ con $f^{\prime} \geq d, \quad v \in R$ y que:

$$
\begin{aligned}
& 2 f^{\prime \prime}+g^{\prime \prime}\left(s_{2}+g^{\prime}-c\right)>0, \text { parav }>0 \\
& 2 f^{\prime \prime}+g^{\prime \prime}\left(s_{2}+g^{\prime}-c\right)<0, \text { para } v<0 \\
& 2 f^{\prime \prime}+g^{\prime \prime}\left(g^{\prime}-c-s_{2}\right)>0, \text { para } v>0 \\
& 2 f^{\prime \prime}+g^{\prime \prime}\left(g^{\prime}-c-s_{2}\right)<0, \text { para } v<0
\end{aligned}
$$

donde $S_{2}=\sqrt{\left(g^{\prime}-c\right)^{2}+4 f^{\prime}}$.
Teorema 1. Para un $\varepsilon>0$, el problema de Cauchy (2), con las condiciones de (1) y suponiendo cumple las condiciones de $A$, tiene solución única global suave $\left(\mathbf{u}^{\varepsilon}(\mathbf{X}, \mathrm{t}), \mathbf{V}^{\varepsilon}(\mathbf{X}, \mathrm{t})\right)$ que satisface

$$
\left|u^{\varepsilon}(x, t)\right| \leq M,\left|v^{\varepsilon}(x, t)\right| \leq M
$$

donde $M$ es una constante positiva independiente de $\boldsymbol{\varepsilon}$.

Demostración: Ver Lu, 2002; Gómez-Plata, 2009.

Una extensión del teorema 1, es la siguiente proposición que extiende el resultado a sistemas elásticos con términos fuentes como en (3) y que será de vital importancia en el estudio de las soluciones débiles de este mismo sistema. Vale aclarar que las estimaciones de soluciones débiles del sistema sin los términos fuentes ya fueron estudiadas por Lu, 2002, más estas estimaciones para el sistema (3), son actualmente materia de estudio e investigación.

Proposición 1. Supongamos que $g_{1}(u, v)$, $g_{2}(u, v)$ cumplen

$w_{u} g_{1}+w_{v} g_{2} \geq c_{1} w+c_{2}, \quad z_{u} g_{1}+z_{v} g_{2} \leq c_{3} w+c_{4}$

donde $C_{1}, C_{2}, C_{3}, c_{4} \in \mathbb{R}$ y $g_{2}=v h(u, v)$ con $\mathrm{h}(\mathrm{u}, \mathrm{v})$ una función continua para $(\mathrm{u}, \mathrm{v})$, entonces el problema de Cauchy (1), (2) tiene única solución $\left(\mathrm{u}^{\varepsilon}(\mathrm{x}, \mathrm{t}), \mathrm{v}^{\varepsilon}(\mathrm{x}, \mathrm{t})\right)$ y satis-face que para todo, $\mathrm{t}>0$, $\left|\mathrm{u}^{\varepsilon}(\mathrm{x}, \mathrm{t})\right| \leq \mathrm{M}(\mathrm{T}) ; 0<\mathrm{c}(\varepsilon, \mathrm{t}) \leq \mathrm{v}^{\varepsilon}(\mathrm{x}, \mathrm{t}) \leq \mathrm{M}(\mathrm{T})$ sobre $\mathbb{R} \times[0, T]$, donde $M(T)$ es una constante positiva que es independiente de $\varepsilon, \mathrm{C}(\varepsilon, \mathrm{t})$ es una función positiva la cual tiende a cero cuando $\varepsilon$ tiende a cero $\circ \boldsymbol{t}$ tiende a infinito.

\section{Demostración:}

El sistema (1) se puede escribir como

$$
\mathrm{U}_{\mathrm{t}}+\mathrm{dFU} \mathrm{x}_{\mathrm{x}}+\mathrm{G}=0
$$




$$
\begin{aligned}
& \text { donde } U=(u, v)^{\top}, F=R^{2} \rightarrow R^{2}, \\
& F:(u, v) a \quad(c u+f(v), u+g(v)) y \\
& d F=\left(\begin{array}{ll}
c & f^{\prime}(v) \\
1 & g^{\prime}(v)
\end{array}\right)
\end{aligned}
$$

para calcular los valores propios de esta ultima matriz $\mathrm{dF}$, se hace $\operatorname{det}(\mathrm{dF}-\lambda \mathrm{I})=0$

de donde

$$
(c-\lambda)\left(g^{\prime}(v)-\lambda\right)-f^{\prime}(v)=0
$$$$
\lambda=\frac{c+g^{\prime} \pm \sqrt{(g-c)^{2}+4 f^{\prime}}}{2}
$$

Ilamando, $s_{2}=\sqrt{\left(g^{\prime}-c\right)^{2}+4 f^{\prime}}$, tenemos que

$$
\lambda_{1}=\frac{\mathrm{c}+\mathrm{g}^{\prime}+\mathrm{S}_{2}}{2}, \lambda_{2}=\frac{\mathrm{c}+\mathrm{g}^{\prime}-\mathrm{S}_{2}}{2}
$$

Los vectores propios a derecha $r_{1}, r_{2}$ asociados se calculan resolviendo

$$
r_{1}=\left[d F-\lambda_{1} l \mid 0\right], \quad r_{2}=\left[d F-\lambda_{2} l \mid 0\right]
$$

de donde obtenemos

$$
r_{1}=\left(g^{\prime}-c+s_{2},-2\right)^{\top}, \quad r_{2}=\left(g^{\prime}-c-s_{2},-2\right)^{\top}
$$

de tal forma que

$$
\begin{aligned}
& \nabla \lambda_{1} \cdot r_{1}=\frac{2 f^{\prime \prime}-g^{\prime \prime}\left(s_{2}-\left(g^{\prime}-c\right)\right)}{S_{2}} ; \\
& \nabla \lambda_{2} \cdot r_{2}=\frac{2 f^{\prime \prime}+g^{\prime \prime}\left(s_{2}+\left(g^{\prime}-c\right)\right)}{S_{2}}
\end{aligned}
$$

de donde los invariantes de Riemman son:

$$
\mathrm{W}=\mathrm{u}+\int_{0}^{\mathrm{v}} \frac{\mathrm{g}^{\prime}-\mathrm{c}+\mathrm{s}_{2}}{2} \mathrm{dv}, \quad \mathrm{z}=\mathrm{u}+\int_{0}^{\mathrm{v}} \frac{\mathrm{g}^{\prime}-\mathrm{c}-\mathrm{s}_{2}}{2} \mathrm{dv}
$$

Calculando

$\mathrm{w}_{v}, \mathrm{w}_{u}, \mathrm{w}_{\mathrm{u}}, \mathrm{w}_{\mathrm{u}}, \mathrm{w}_{\mathrm{w}} ; \mathrm{z}_{v}, \mathrm{z}_{\mathrm{u}}, \mathrm{z}_{\mathrm{uu}}, \mathrm{z}_{\mathrm{uv}}, \mathrm{z}_{\mathrm{w}}$; tenemos

$$
\begin{aligned}
& w_{u}=1+\frac{\partial}{\partial u} \int_{0}^{v} \frac{g^{\prime}-c+s_{2}}{2} d v=1 \\
& z_{u}=1+\frac{\partial}{\partial u} \int_{0}^{v} \frac{g^{\prime}-c-s_{2}}{2} d v=1
\end{aligned}
$$

$w_{v}=1+\frac{\partial}{\partial v} \int_{0}^{v} \frac{g^{\prime}-c+s_{2}}{2} d v=\frac{g^{\prime}-c+s_{2}}{2}$,

$\mathrm{z}_{\mathrm{v}}=1+\frac{\partial}{\partial \mathrm{u}} \int_{0}^{\mathrm{v}} \frac{\mathrm{g}^{\prime}-\mathrm{c}-\mathrm{s}_{2}}{2} d v=\frac{g^{\prime}-\mathrm{c}-\mathrm{s}_{2}}{2}$

$w_{w}=\frac{g^{\prime \prime}\left(g^{\prime}-c+s_{2}\right)+2 f^{\prime \prime}}{2 s_{2}}$,

$z_{w}=\frac{2 f^{\prime \prime}+g^{\prime \prime}\left(g^{\prime}-c-s_{2}\right)}{2 s_{2}}$

$w_{u U}=0, \quad w_{u_{N}}=0, z_{u U}=0, z_{v}=0$

Multiplicando el sistema (3) por

$\nabla \mathrm{z}(\mathrm{u}, \mathrm{t})=\left(\mathrm{z}_{\mathrm{u}}, \mathrm{z}_{\mathrm{v}}\right)$, tenemos

$\nabla \mathrm{z}\left(\mathrm{U}_{\mathrm{t}}+\mathrm{dFU} \mathrm{U}_{\mathrm{x}}+\mathrm{G}\right)=\varepsilon \Delta \mathrm{U}$

si $\nabla \mathbf{Z d F}=\lambda_{1} \nabla \mathbf{z}$, encontramos

$z_{t}+\lambda_{1} z_{x}+\left(z_{u} g_{1}+z_{v} g_{2}\right)=$

$\nabla \mathrm{z} \varepsilon \Delta \mathrm{U}=\varepsilon\left(\mathrm{Z}_{\mathrm{u}}, \mathrm{Z}_{v}\right)\left(\mathrm{u}_{x x}, \mathrm{v}_{\mathrm{xx}}\right)=$

$\varepsilon \mathrm{z}_{x x}-\varepsilon\left(\mathrm{z}_{\mathrm{uu}} \mathrm{u}_{\mathrm{x}}^{2}+2 \mathrm{z}_{\mathrm{u}} \mathrm{u}_{\mathrm{x}} \mathrm{v}_{\mathrm{x}}+\mathrm{z}_{\mathrm{w}} \mathrm{v}_{\mathrm{x}}^{2}\right)$ 
remplazando $\mathbf{Z}_{\mathrm{u}}, \mathbf{Z}_{\mathrm{uN}}, \mathbf{Z}_{\mathrm{w}}$ encontramos

$$
\begin{aligned}
& z_{t}+\lambda_{1} z_{x}+\left(z_{u} g_{1}+z_{v} g_{2}\right)=\varepsilon z_{x x}-\varepsilon\left(z_{w}\right) v_{x}^{2}= \\
& \varepsilon z_{x x}-\varepsilon\left(\frac{2 f^{\prime \prime}+g^{\prime \prime}\left(g^{\prime}-c-s_{2}\right)}{S_{2}}\right) v_{x}^{2}
\end{aligned}
$$

por $A$, si $V>0,2 f^{\prime \prime}+g^{\prime \prime}\left(g^{\prime}-c-s_{2}\right)>0$, entonces

$$
z_{\mathrm{t}}+\lambda_{1} \mathrm{z}_{\mathrm{x}}+\left(\mathrm{z}_{\mathrm{u}} \mathrm{g}_{1}+\mathrm{z}_{\mathrm{v}} g_{2}\right) \leq \varepsilon \mathrm{z}_{x x}
$$

por $A$, si $V<0, \quad 2 f^{\prime \prime}+g^{\prime \prime}\left(g^{\prime}-C-s_{2}\right)<0$, entonces

$z_{t}+\lambda_{1} z_{x}+\left(z_{u} g_{1}+z_{v} g_{2}\right) \geq \varepsilon z_{x x} \quad(6)$

\section{Multiplicando el sistema (3) por}

$$
\nabla w(u, t)=\left(w_{u}, w_{v}\right) \text {, }
$$

tenemos

$$
\nabla \mathrm{w}\left(\mathrm{U}_{\mathrm{t}}+\mathrm{dF} \mathrm{U}_{\mathrm{x}}+\mathrm{G}\right)=\varepsilon \Delta \mathrm{U}
$$

$$
\text { si } \nabla z \mathrm{dF}=\lambda_{1} \nabla \mathbf{z} \text {, }
$$

encontramos

$$
\begin{gathered}
\mathrm{w}_{\mathrm{t}}+\lambda_{2} \mathrm{w}_{\mathrm{x}}=\nabla \mathrm{w} \varepsilon \Delta \mathrm{U}=\varepsilon\left(\mathrm{w}_{\mathrm{u}}, \mathrm{w}_{\mathrm{u}}\right)\left(\mathrm{u}_{\mathrm{xx}}, \mathrm{v}_{\mathrm{xx}}\right)= \\
\varepsilon \mathrm{w}_{\mathrm{xx}}-\varepsilon\left(\mathrm{w}_{\mathrm{uu}} \mathrm{u}_{\mathrm{x}}^{2}+2 \mathrm{w}_{\mathrm{uv}} \mathrm{u}_{\mathrm{x}} \mathrm{v}_{\mathrm{x}}+\mathrm{w}_{\mathrm{w}} \mathrm{v}_{\mathrm{x}}^{2}\right)
\end{gathered}
$$

remplazando $\mathbf{W}_{\mathbf{u}}, \mathrm{W}_{\mathrm{w}}, \mathrm{W}_{\mathrm{w}}$ encontramos

$$
\begin{gathered}
\mathrm{w}_{\mathrm{t}}+\lambda_{2} \mathrm{w}_{\mathrm{x}}+\left(\mathrm{w}_{\mathrm{u}} \mathrm{g}_{1}+\mathrm{w}_{\mathrm{v}} \mathrm{g}_{2}\right)=\varepsilon \mathrm{w}_{x x}-\varepsilon\left(\mathrm{w}_{\mathrm{w}}\right) \mathrm{v}_{\mathrm{x}}^{2}= \\
\varepsilon \mathrm{w}_{x \mathrm{x}}-\varepsilon\left(\frac{2 \mathrm{f}^{\prime \prime}+\mathrm{g}^{\prime \prime}\left(\mathrm{g}^{\prime}-\mathrm{C}+\mathrm{S}_{2}\right)}{\mathrm{S}_{2}}\right) \mathrm{v}_{\mathrm{x}}^{2}
\end{gathered}
$$

por $A$, si $V>0, \quad 2 f^{\prime \prime}+g^{\prime \prime}\left(g^{\prime}-C+S_{2}\right)>0$,

entonces

$w_{t}+\lambda_{2} w_{x}+\left(w_{u} g_{1}+w_{v} g_{2}\right) \leq \varepsilon z_{x x}$

por A, si $V<0,2 f^{\prime \prime}+g^{\prime \prime}\left(g^{\prime}-c+s_{2}\right)<0$, entonces

$\mathrm{w}_{\mathrm{t}}+\lambda_{2} \mathrm{w}_{\mathrm{x}}+\left(\mathrm{w}_{\mathrm{u}} \mathrm{g}_{1}+\mathrm{w}_{\mathrm{v}} \mathrm{g}_{2}\right) \geq \varepsilon \mathrm{w}_{\mathrm{xx}} \quad$ (8)

Tomemos (5) y (7) así

$$
\left(-z_{t}\right)+\lambda_{1}\left(-z_{x}\right)+\left(z_{u} g_{1}+z_{v} g_{2}\right) \geq \varepsilon\left(-z_{x \alpha}\right)(9)
$$

$w_{t}+\lambda_{2} w_{x}+\left(w_{u} g_{1}+w_{v} g_{2}\right) \leq \varepsilon z_{x x}$

y (6), (8) así

$$
\begin{aligned}
& z_{t}+\lambda_{1} z_{x}+\left(z_{u} g_{1}+z_{v} g_{2}\right) \geq \varepsilon z_{x x} \\
& -w_{t}-\lambda_{2} w_{x}-\left(w_{u} g_{1}+w_{v} g_{2}\right) \leq-\varepsilon w_{x x}
\end{aligned}
$$

Al considerar las desigualdades (9), (10) y (11),(12) con variables en $\mathbf{W}, \mathbf{Z}$ y aplicando el corolario 1 de Gómez-Plata, 2012, vemos que $\mathrm{W}\left(\mathrm{U}^{\varepsilon}, \mathrm{V}^{\varepsilon}\right) \leq \mathrm{N}(\mathrm{T}),-\mathrm{Z}\left(\mathrm{U}^{\varepsilon}, \mathrm{V}^{\varepsilon}\right) \leq-\mathrm{N}(\mathrm{T})$ sobre $\mathbb{R} \times[0, T]$ donde $\mathrm{N}(\mathrm{T})$ es independiente de $\varepsilon$ y $T \in \mathbb{R}^{+}$y se puede encontrar un $\mathrm{M}(\mathrm{T})>0$ tal que $\left|\mathbf{u}^{\varepsilon}(\mathrm{x}, \mathrm{t})\right| \leq \mathrm{M},\left|\mathbf{V}^{\varepsilon}(\mathrm{x}, \mathrm{t})\right| \leq \mathrm{M}(\mathrm{T})$; del teorema 5 y del lema 4 en Gómez-Plata, 2012, se tiene que $0<\mathrm{C}(\varepsilon, \mathrm{t}) \leq \mathrm{V}^{\varepsilon}(\mathrm{x}, \mathrm{t}) \leq \mathrm{M}(\mathrm{T})$ 
Es muy importante tener en cuenta que gracias a la flexibilidad de la metodología usada en este artículo es posible aplicarla para resolver otros sistemas de ecuaciones diferenciales parciales que contienen leyes de conservación hiperbólica, por ejemplo se pueden llevar a sistemas de Euler, de Roux, de gases y algunos otros que bajo ciertas condiciones puede aplicarsele el principio del máximo.

\section{ASPECTOS DE DISCUSION}

Los sistemas de elasticidad de la forma

$$
\left\{\begin{array}{l}
u_{t}+(c u+f(v))_{x}+g_{1}(u, v)=0 \\
v_{t}+(u+g(v))_{x}+g_{2}(u, v)=0
\end{array}\right.
$$

tiene estimaciones viscosas de sus soluciones globales que son suaves; bajo la metodología de compacidad compensada se pueden estimar soluciones débiles de este mismo sistema, teniendo en cuenta la proposición 1, demostrada en este artículo y que es una extensión del teorema 1, que generaliza este teorema a sistemas de elasticidad con término fuente. La idea en este caso es usar las estimaciones encontradas para este sistema, guiados por el trabajo de Lu, 2002 y siguiendo los resultados que Yan et al, 2007.

Es muy importante tener en cuenta que gracias a la flexibilidad de la metodología usada en este artículo es posible aplicarla para resolver otros sistemas de ecuaciones diferenciales parciales que contienen leyes de conservación hiperbólica, por ejemplo se pueden llevar a sistemas de Euler, de Roux, de gases y algunos otros que bajo ciertas condiciones puede aplicarsele el principio del máximo. La metodología paralela de regiones invariantes aporta un camino por donde se puede orientar el interesado para escoger sistemas de leyes de conservación que posiblemente se les pueda aplicar el principio del máximo.

\section{AGRADECIMIENTOS}

El desarrollo del presente trabajo fue posible gracias al apoyo de la Facultad de Ciencias Básicas y Aplicadas de la UMNG y, al entonces Decano, Dr. Fernando Cantor Rincón, quien apoyó al autor para cursar su maestría en Matemáticas Aplicadas y realizar el proyecto de investigación en Leyes de conservación Hiperbólica; de estos trabajos nace el resultado de este artículo. 


\section{REFERENCIAS}

1. Ciarlet P.G.1988. Mathematical Elasticity, tree-dimensional elasticity of studies in mathematics and its aplications.Vol 20, North-Holland Publishing Co, Amsterdam. 650p.

2. Evans L. 1998. Partial differential equation. Primera Edición. American Mathematical Society. Berkeley. 741 p.

3. Gómez-Plata A.R. 2009. Solución a sistemas de elasticidad sin Fuente. Revista Facultad de Ciencias Básicas. 5:44-49.

4. Gómez-Plata A.R. 2012. Solución a un sistema de elasticidad generalizado, tesis de Maestría en Matemática Aplicada, Universidad EAFIT. Medellin, 65p.

5. Gurtin M.E.1981. Topics in Finite Elasticity. Society for Industrial Applied Mathematics. Philadelphia, Penssylvania. 58p.

6. Landau L., Lifshits M. 1985. Mecánica I. Tomo I, Editorial Reverte, Barcelona. 201p.

7. Landis E. 1997. Second order equations of elliptic and parabolic type. Vol 171. American Mathematical Society. Berkeley. 203 p.

8. Lu Y. 2002. Hyperbolic conservations Laws and the compensated compacts method. Vol 128, Chapman and Hall, New York. 239 p.

9. Protter M., Weinberger, H. 1999. Maximun principles in Differential Equations. Springer. New York. 257 p.

10. Yan J., Zhixin C., Ming T. 2007a. Conservations Laws I: Viscosity Solutions. Revista Colombiana de Matemáticas, 41:81-90.

11. Yan J., Zhixin C., Ming T. 2007b. Conservations Laws II: Weak Solutions, Revista Colombiana de Matemáticas, 41:91-106. 